\title{
Transient NK Cell Depletion Facilitates Pulmonary Osteosarcoma Metastases After Intravenous Inoculation in Athymic Mice
}

\author{
Michael A. Harris, BBioMed Hons, ${ }^{1}$ Tanmay M. Shekhar, PhD, ${ }^{1}$ Lucy A. Coupland, PhD, ${ }^{2}$ \\ Mark A. Miles, $\mathrm{PhD},{ }^{1}$ and Christine J. Hawkins, $\mathrm{PhD}^{1}$
}

Two thirds of metastatic osteosarcoma patients die within 5 years of diagnosis. Improved experimental models of osteosarcoma metastasis will facilitate the development of more effective therapies. Intravenous cancer cell injection can produce lung metastases in nude mice, but this "experimental metastasis" technique has been predominantly applied to a single osteosarcoma cell line (143B) and required injection of 1-2 million cells. Using two human osteosarcoma cell lines, we discovered that transient Natural Killer cell depletion dramatically enhanced the efficiency of experimental pulmonary osteosarcoma metastasis. This technique for modeling osteosarcoma metastasis may enable the identification of better treatments for this aggressive cancer.

Keywords: osteosarcoma, bone cancer, xenograft, metastasis, Natural Killer cells

\section{Introduction}

O STEOSARCOMA, THE MOST common type of primary bone cancer, is most frequently diagnosed in children and teenagers. ${ }^{1}$ Patients with pulmonary osteosarcoma metastases have a dire prognosis, with less than a third surviving for more than 5 years $^{2}$; thus, more effective treatments are urgently required. Animal models are essential for the identification of promising new therapies for clinical evaluation.

Mice genetically engineered to spontaneously develop osteosarcomas and large dog breeds inherently susceptible to osteosarcoma offer advantages of natural tumor development and metastasis, as well as inter-individual variability. ${ }^{3}$ However, the logistics involved in detecting tumors that arise stochastically over many months or years renders these models economically and temporally unsuitable for initial in vivo assessments of possible new treatments. Introduction of human or rodent osteosarcoma cell lines into immuno-deficient or competent mice or rats provides predictable kinetics and reproducible tumor behavior, so although these models bypass the initial steps of tumor formation, they are more feasible than spontaneous models for determining the potential efficacy of new therapies.

Several human osteosarcoma cell lines have been previously reported to spontaneously metastasize to mouse lungs, after subcutaneous, intramuscular, intrafemoral, or intratibial implantation. However, to evaluate the ability of candidate new drugs to target established metastases, which represents the most urgent clinical need, the presence of a primary tumor in these models can present major challenges. For example, if the drug being tested has little effect on the primary tumor, the mouse may require ethical culling before an effect on lung metastases can be detected. If the drug does possess activity against the primary tumor, it can be difficult to determine whether treatment-associated reductions in lung metastasis result from impaired dissemination from the primary tumor or destruction of the osteosarcoma cells growing within the lungs. Hence, to assess the activity of drugs against established osteosarcoma metastases, it is advantageous to use mice bearing established pulmonary osteosarcoma metastases in the absence of primary tumors.

Intravenous injection of tumor cell lines is a popular method for experimentally generating lung metastases of numerous cancer types in mice lacking primary tumors. ${ }^{4}$ The application to osteosarcoma xenografts in nude mice, however, has been limited. To our knowledge, only one human osteosarcoma cell line, 143B, has been used by multiple groups to induce so-called "experimental metastasis." ${ }^{-9}$ Those studies involved injection of at least one million cells to achieve lung metastases. The dearth of examples of osteosarcoma cell lines published to form metastases after intravenous injection, coupled with the large number of $143 \mathrm{~B}$ cells required to achieve this, implies that the process by which human osteosarcoma cells move from the blood to the lungs in nude mice is extremely inefficient.

Natural Killer (NK) cells are innate immune cells that recognize and destroy cells with an abnormal expression profile of

\footnotetext{
${ }^{1}$ Department of Biochemistry and Genetics, La Trobe Institute for Molecular Science, La Trobe University, Bundoora, Australia.

${ }^{2}$ The ACRF Department of Cancer Biology and Therapeutics, The John Curtin School of Medical Research, The Australian National University, Canberra, Australia.
} 
specific surface receptors such as major histocompatibility complex (MHC) Class I and NKG2D ligands. These proteins are down- and upregulated, respectively, in virally infected or cancerous cells, thus triggering destruction by NK cells. ${ }^{10}$ There is substantial evidence that NK cells help protect against the development and spread of cancers, including osteosarcoma in humans, ${ }^{11}$ but their numbers and function are significantly reduced in patients with more aggressive disease ${ }^{12}$; thus, treatments that reverse these effects are being actively pursued. ${ }^{13} \mathrm{NK}$ cells impair the tumorigenicity of some human cancer cells on introduction into mice lacking adaptive immune cells, ${ }^{14}$ and they have been shown to impede lung metastasis by up to $95 \%$ in immunocompetent mice. ${ }^{15,16}$

In this study, we tested the hypothesis that NK cellmediated destruction of human osteosarcoma cells limits their ability to reach the lungs of nude mice, extravasate, and form metastases there after intravenous injection. We reasoned that this could be especially significant in BALB/c nu/ $n u$ mice, which possess $50 \%$ more NK cells than their immunocompetent counterparts. ${ }^{17}$

\section{Methods}

The experiments performed in this study were approved by the La Trobe Animal Ethics Committee (AEC17-76), and they complied with the Australian Code of Practice for the Care and Use of Animals for Scientific Purposes. BALB/c-Foxn $1^{\text {nu }} /$ Arc ("nude") mice were purchased from the Animal Resource Centre (Canning Vale, WA, Australia); transported to the La Trobe Animal Research and Training Facility, where they were housed in ventilated cages with 12 hours light/dark cycling and unrestricted access to food and water; and subjected to daily weighing and monitoring. A week after transfer, the mice were injected intraperitoneally with $50 \mu \mathrm{L}$ of normal saline or anti-asialo-GM1 (Wako, Osaka, Japan).

For NK cell monitoring, blood (collected 1, 3, 7, or 14 days later via submandibular bleeding) was mixed with a 10 -fold volume excess of filter-sterilized red cell lysis buffer $(0.15 \mathrm{M}$ ammonium chloride, $10 \mathrm{mM}$ potassium bicarbonate, and $0.1 \mathrm{mM}$ ethylenediaminetetraacetic acid [EDTA]) for 10 minutes at room temperature, washed in RPMI 1640 with HEPES (ThermoFisher Scientific, Waltham, MA), and finally resuspended in phosphate-buffered saline supplemented with $4 \%$ fetal bovine serum and $1 \mathrm{mM}$ EDTA containing either antibodies diluted 1/300 recognizing cell surface markers: F4/ 80-PE-Cy7 clone BM8 \#25-4801-82 (ThermoFisher Scientific), Ly-6G-APC clone 1A8 \#560599 (BD Biosciences, Franklin Lakes, NJ), and CD335/NKP46-BV421 clone 29A1.4 \#562850 (BD Biosciences), or a panel of isotype control antibodies bearing the same fluorophores, sourced from the same companies (\#25-4321-82; \#553932; \#562602).

For assessing metastases, luciferase-tagged KRIB or 143B cell lines ${ }^{18}$ were injected into the lateral tail vein of mice 1 day after administration of saline or anti-asialo-GM1. Each week for 4 weeks, the mice were injected intraperitoneally with $150 \mathrm{mg} / \mathrm{kg}$ of D-luciferin (Pure Science, Porirua, New Zealand) and anesthetized by using isoflurane; an IVIS Lumina XR III (Perkin Elmer, Waltham, MA) was used to measure luminescence each minute over 10 minutes with automatic exposure times. The highest luminescence (photons per second) within a region of interest corresponding to lung tumors was recorded. For presenting comparative images, a uniform intensity scale was used. All images belonging to one experiment were loaded as a group by using the living image 4.7 software (Perkin Elmer), and the minimum intensity increased until all background luminescence was no longer visible. The maximum intensity was decreased until the most intense metastasis was reflected as the peak value on the scale. Mice were culled by carbon dioxide asphyxiation at the end of the experiment, or earlier if tumor-related symptoms met ethically determined criteria, including weight loss, respiratory distress or decreased activity, alertness, or movement.

For histology, lungs were incubated in formalin, $70 \%$ ethanol, $100 \%$ ethanol, and then xylene; then paraffin-embedded, sectioned, and finally stained with hematoxylin and eosin. ${ }^{19}$

\section{Results}

Our initial aim was to establish an experimental lung metastasis model using the KRIB human osteosarcoma cell line in nude mice, to evaluate the efficacy of drugs against established pulmonary osteosarcoma metastases. KRIB cells have been reported to metastasize from intratibial tumors to the lungs, ${ }^{20}$ and we previously observed that intramuscular implantations of luciferase-tagged KRIB human osteosarcoma cells (KRIB-luc) into nude mice invariably led to pulmonary metastases. ${ }^{18}$ Those studies demonstrated that these cells thrived within the microenvironment of nude mouse lungs. We were surprised, therefore, that 4 weeks after tail vein injection of 150,000 KRIB-luc cells, no bioluminescence signal could be detected in the animals' lungs.

To test the hypothesis that NK cells may have eliminated the KRIB-luc cells before they were able to form lung metastases, we repeated the experiment but administered an anti-asialoGM1 antibody ${ }^{21}$ to deplete NK cells 1 day before tumor cell injection. The percentage of circulating NK cells dropped on average from $10 \%$ to $0.6 \%$ the day after treatment, with NK cell numbers recovering a week later (Fig. 1). This temporary NK depletion substantially affected the ability of osteosarcoma cells to form pulmonary metastases. Intravenous injection with 150,000 KRIB-luc cells 1 day after anti-asialo-GM1 administration produced lung metastases in the majority of mice (Fig. 2). In subsequent experiments, 45 out of 64 mice (70\%) developed lung metastases after receiving tail vein injections of 150,000 KRIB-luc cells, 1 day after NK depletion (data not shown).

We next investigated whether transient NK cell depletion increased the efficiency with which intravenous inoculation of 143B human osteosarcoma cells produced lung metastases. As mentioned earlier, previous research established that these cells could form lung metastases after intravenous injection, ${ }^{5-9}$ but 1-2 million cells were injected in those studies, implying that seeding to the lungs was very inefficient. As with the KRIB cells, tail vein injections of 150,000 luciferase-tagged 143B cells failed to yield lung metastases (Fig. 3). However, injection of 150,000 143B cells the day after NK depletion produced extremely aggressive metastases in the lungs (and other organs such as the liver), which necessitated euthanasia between 15 and 27 days after cell inoculation (average survival was 18.6 days). With the aim of developing a less aggressive model that would enable testing of drug efficacy, we reduced the number of $143 \mathrm{~B}$ cells that we injected from 150,000 to 50,000 cells. Mice that received the lower number of 143B cells the day after NK depletion also all developed lung metastases, but they survived somewhat longer. Those mice were 

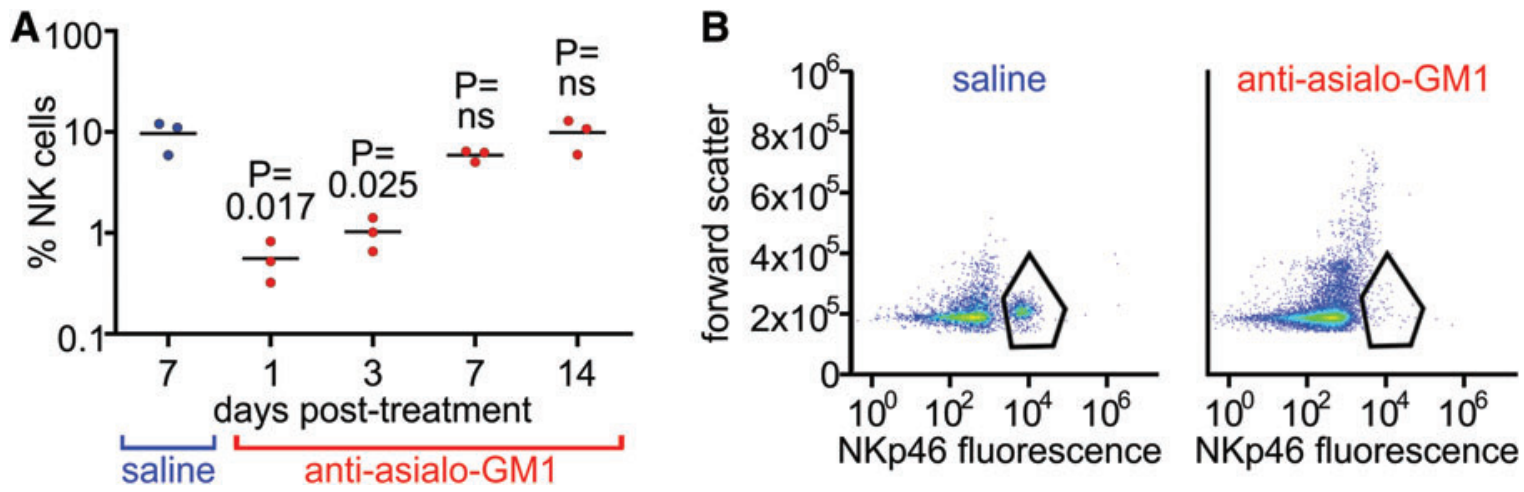

FIG. 1. Anti-asialo-GM1 treatment transiently depletes circulating NK cells. (A) Blood was harvested 1, 3, 7, or 14 days after treatment of nude mice with anti-asialo-GM1, or 7 days after saline administration. After red cell lysis, the proportion of peripheral blood mononuclear cells that were NK cells (designated as Ly6G-, F4/80-, NKp46+) were quantitated by flow cytometry. One-way ANOVAs with Dunnett's adjustments for multiple comparisons were performed to compare the abundance of NK cells at each timepoint after anti-asialo-GM1 treatment, relative to saline-treated mice. (B) Examples of flow cytometry data are shown from blood samples from mice injected with saline, or 1 day after administration with antiasialo-GM1. ANOVA, analysis of variance; NK, Natural Killer. Color images are available online.

euthanized 16-42 days after cell inoculation, with an average survival of 26 days (data not shown).

\section{Discussion}

These data reveal that cells targeted by the anti-asialo-GM1 antibody reduce the establishment of human osteosarcoma lung metastases in nude mice. This antibody was developed to deplete NK cells, ${ }^{21}$ but it was subsequently also demonstrated to temporarily reduce basophil numbers, ${ }^{22}$ leaving other leukocyte lineages unaffected. ${ }^{22,23}$ We cannot formally exclude the possibility that basophil ablation contributed to the pro-metastatic effect of anti-asialo-GM1 treatment we observed, but the distinct function of these cells implies that NK depletion was the

FIG. 2. NK depletion facilitates lung metastases after intravenous inoculation with luciferase-expressing KRIB osteosarcoma cells. Nude mice were intravenously inoculated with 150,000 luciferaseexpressing human KRIB osteosarcoma cells, a day after treatment with saline or anti-asialo-GM1. Bioluminescence was detected weekly. (A) Images of each mouse are shown, with luminescence indicated on a color scale. (B) Quantitation of lung signals was graphed. (C) Lung tissue from a mouse that received KRIB-luc cells after NK depletion was subjected to hematoxylin and eosin staining. The bar represents $100 \mu \mathrm{m}$. Color images are available online.
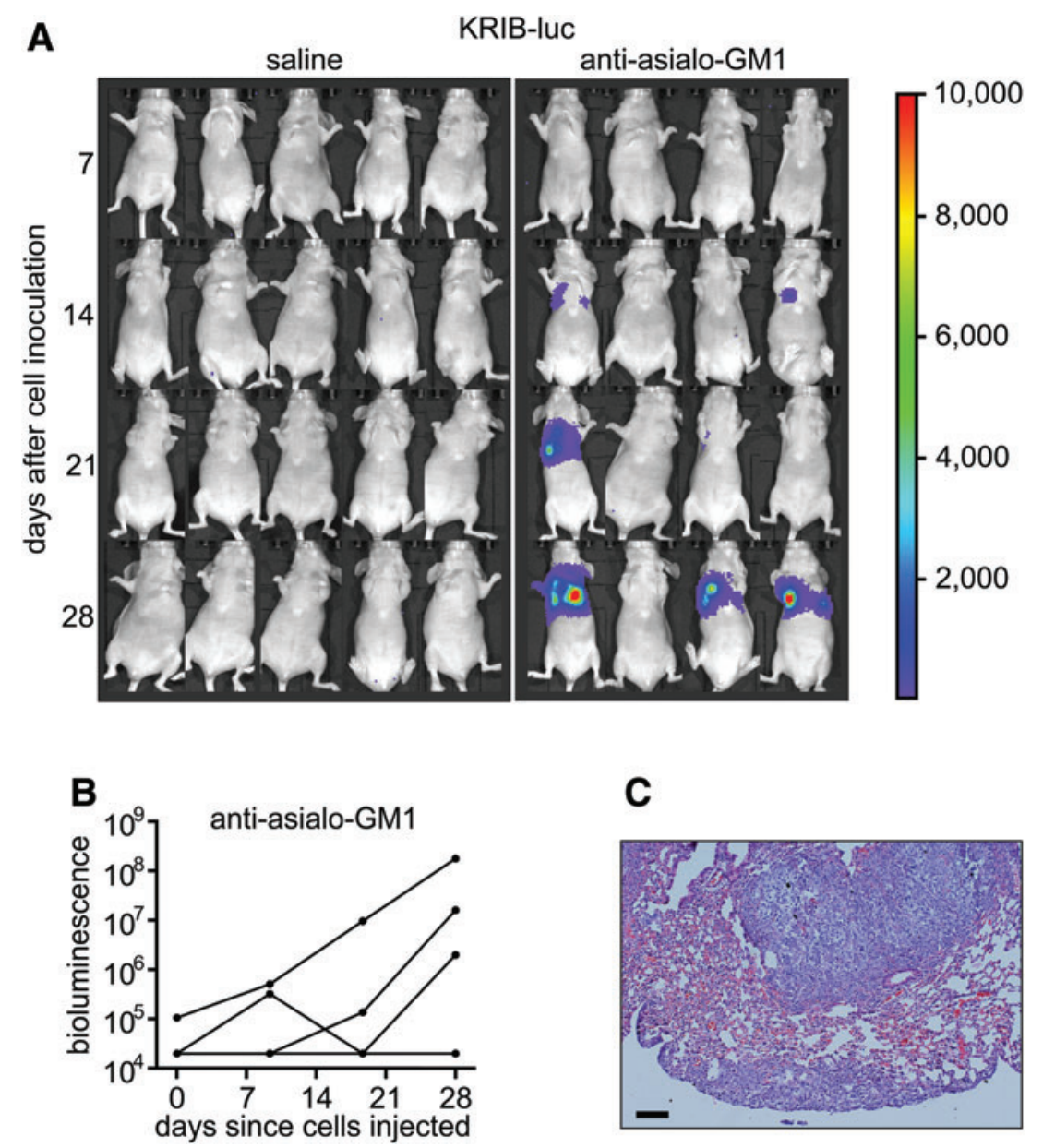

C

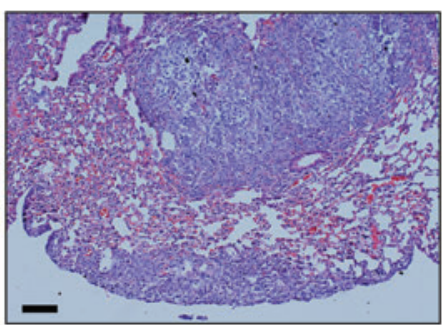



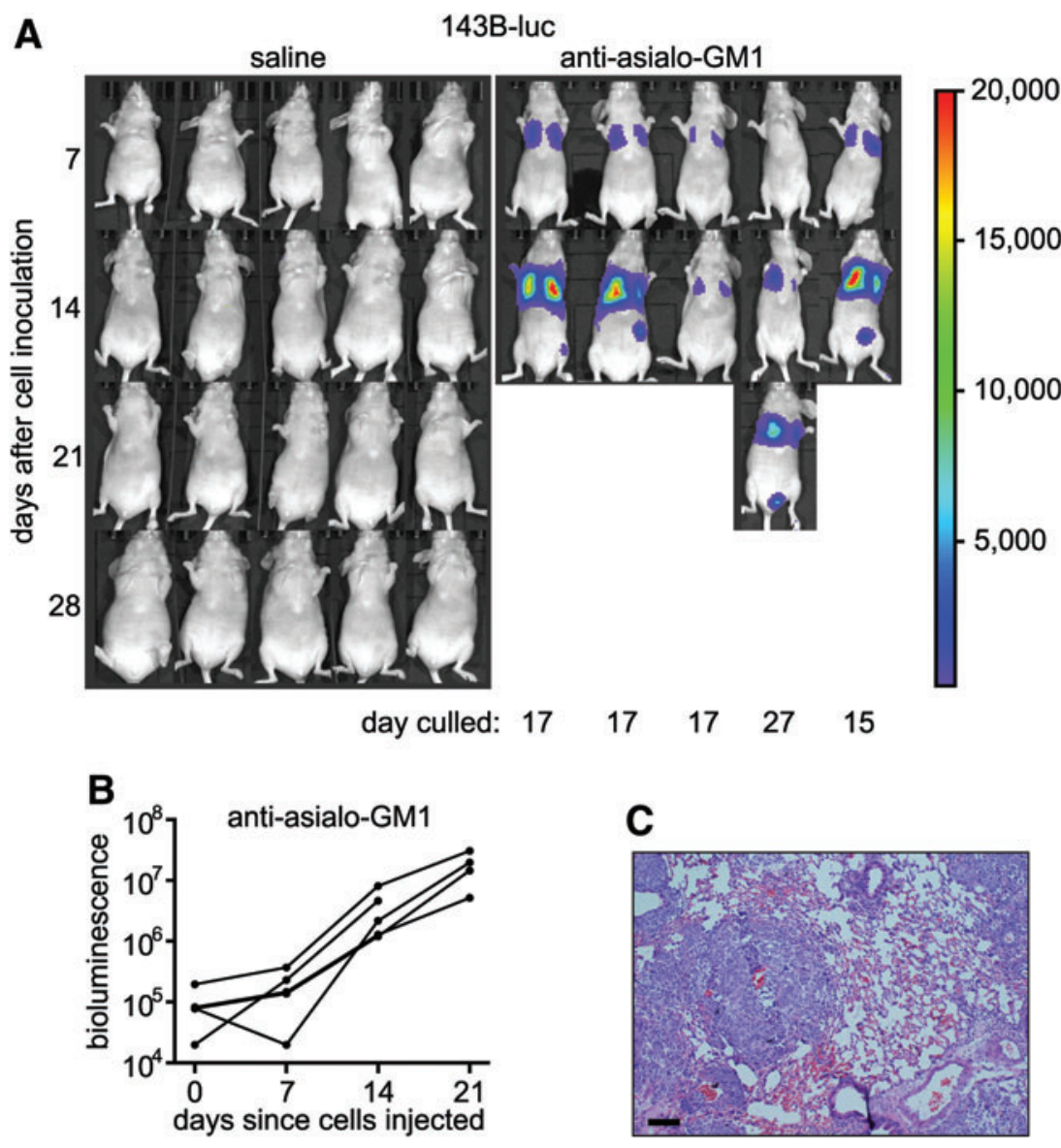

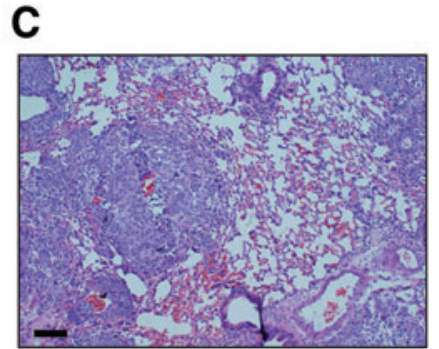

FIG. 3. NK depletion facilitates lung metastases after intravenous inoculation with luciferase-expressing 143B osteosarcoma cells. Nude mice were intravenously inoculated with 150,000 luciferaseexpressing human 143B osteosarcoma cells, a day after treatment with saline or anti-asialo-GM1. Bioluminescence was detected weekly. (A) Images of each mouse are shown, with luminescence indicated on a color scale. Mice that were inoculated with 143B cells after receiving anti-asialo-GM1 were euthanized on the indicated days due to metastasis-related symptoms. (B) Quantitation of lung signals was graphed. (C) Lung tissue from a mouse that received 143B-luc cells after NK depletion was subjected to hematoxylin and eosin staining. The bar represents $100 \mu \mathrm{m}$. Color images are available online. primary mechanism through which the antibody enabled formation of metastases. NK cells are well established to destroy cancer cells, including osteosarcoma cells, ${ }^{11}$ whereas basophils drive IgE-induced allergic inflammation. ${ }^{24}$ Transient NK depletion permitted the formation of lung metastases, which continued to grow at a consistent rate even weeks after NK levels were restored, consistent with published observations that NK cells failed to penetrate into pulmonary osteosarcoma me$\operatorname{tastases}^{25}$ and that anti-asialo-GM1 treatment promoted experimental melanoma metastasis when administered before cell inoculation or 1 hour afterward, but had markedly reduced impact if given 3 or more hours later. ${ }^{23}$ These results highlight the ability of NK cells to destroy osteosarcoma cells en route to the lungs, but they reinforce the notion that they have negligible impact on established metastases.

Lung metastasis was reported to result from intravenous injection of as few as 20,000 murine osteosarcoma cells into syngeneic immunocompetent mice, ${ }^{26}$ whereas experimental metastases models involving intravenous injection of human osteosarcoma cells into nude mice invariably required inoculation with at least a million cells. ${ }^{5-8,27,28}$ In this study, a temporary reduction in NK cells within nude mice massively enhanced the efficiency of metastasis. Presumably, the enhanced recognition by murine NK cells of non-murine osteosarcoma cells, coupled with a higher number of circulating NK cells in nude mice, contributes to the inefficiency with which intravenously injected human osteosarcoma cells form lung metastases in these mice.

We suggest that this model of NK cell depletion before intravenous inoculation of osteosarcoma cells into nude mice may enable the development of additional osteosarcoma metastasis models and will hopefully progress research toward more effective therapies for patients with this lethal disease. Once NK cells rebound to normal levels, such models are minimally immunodeficient; hence, they would recapitulate tumor responses in patients more faithfully than models using more severely immunodeficient animals.

Although this study focused on experimental metastases derived from established human osteosarcoma cell lines, we hope this approach may also enable the development of patientderived xenograft (PDX) models of osteosarcoma metastasis. To date, osteosarcoma PDX models have modeled primary tumors, through heterotypic ${ }^{29}$ or orthotopic ${ }^{30}$ introduction of tumor samples into immunocompromised mice. Intravenous inoculation of patient-derived cancer samples into severely immunodeficient mice has, however, been used to develop PDX models of pulmonary breast cancer metastases. ${ }^{31,32}$ Hopefully, the NK depletion method described here could enable similar PDX models of pulmonary osteosarcoma metastases to be developed in minimally immunodeficient animals.

\section{Acknowledgments}

The authors thank the staff from the La Trobe Animal Research and Training Facility for assistance with animal experiments, and Peter Lock and the Bioimaging Platform for help with microscopy and flow cytometry.

\section{Author Disclosure Statement}

No competing financial interests exist. 


\section{Funding Information}

This study was funded by grants from The Kids' Cancer Project, Tour de Cure, and a Grant-in-Aid from the Cancer Council Victoria.

\section{References}

1. Mirabello L, Troisi RJ, Savage SA. Osteosarcoma incidence and survival rates from 1973 to 2004: data from the Surveillance, Epidemiology, and End Results Program. Cancer. 2009;115(7):1531-43.

2. Meazza C, Scanagatta P. Metastatic osteosarcoma: a challenging multidisciplinary treatment. Expert Rev Anticancer Ther. 2016;16(5):543-56.

3. Fan TM. Animal models of osteosarcoma. Expert Rev Anticancer Ther. 2010;10(8):1327-38.

4. Price JE. Spontaneous and experimental metastasis models: nude mice. Methods Mol Biol (Clifton, NJ). 2014;1070:223-33.

5. Klangjorhor J, Chaiyawat $\mathrm{P}$, Teeyakasem $\mathrm{P}$, et al. Mycophenolic acid is a drug with the potential to be repurposed for suppressing tumor growth and metastasis in osteosarcoma treatment. Int J Cancer. 2019 [Epub ahead of print]; DOI: $10.1002 / \mathrm{ijc} .32735$.

6. Lu K-H, Chen P-N, Hsieh Y-H, et al. 3-Hydroxyflavone inhibits human osteosarcoma U2OS and 143B cells metastasis by affecting EMT and repressing u-PA/MMP-2 via FAK-Src to MEK/ERK and RhoA/MLC2 pathways and reduces 143B tumor growth in vivo. Food Chem Toxicol. 2016;97:177-86.

7. Tome $\mathrm{Y}$, Yano $\mathrm{S}$, Sugimoto $\mathrm{N}$, et al. Use of $\alpha \mathrm{v}$ integrin linked to green fluorescent protein in osteosarcoma cells and confocal microscopy to image molecular dynamics during lung metastasis in nude mice. Anticancer Res. 2016;36(8):3811-6.

8. Tome Y, Kimura H, Maehara H, et al. High lung-metastatic variant of human osteosarcoma cells, selected by passage of lung metastasis in nude mice, is associated with increased expression of $\alpha(\mathrm{v}) \beta(3)$ integrin. Anticancer Res. 2013; 33(9):3623-7.

9. Igarashi K, Kawaguchi K, Kiyuna T, et al. Antimetastatic efficacy of the combination of caffeine and valproic acid on an orthotopic human osteosarcoma cell line model in nude mice. Anticancer Res. 2017;37(3):1005-11.

10. Prager I, Watzl C. Mechanisms of natural killer cell-mediated cellular cytotoxicity. J Leukoc Biol. 2019;105(6):1319-29.

11. Tarek N, Lee DA. Natural killer cells for osteosarcoma. Adv Exp Med Biol. 2014;804:341-53.

12. Sutlu T, Alici E. Natural killer cell-based immunotherapy in cancer: current insights and future prospects. J Intern Med. 2009;266(2):154-81.

13. Sun H, Sun C. The rise of NK cell checkpoints as promising therapeutic targets in cancer immunotherapy. Front Immunol. 2019;10:2354.

14. Okada S, Vaeteewoottacharn K, Kariya R. Application of highly immunocompromised mice for the establishment of patient-derived xenograft (PDX) models. Cells. 2019;8(8):889.

15. Coupland LA, Chong BH, Parish CR. Beware of NK cells in pre-clinical metastasis models. Clin Exp Metastasis. 2013;30(7):945-7.

16. Isaacson B, Mandelboim O. Natural killer cells control metastasis via structural editing of primary tumors in mice. Cancer Immunol Immunother. 2019;68(10):1721-4.

17. Kariya R, Matsuda K, Gotoh K, et al. Establishment of nude mice with complete loss of lymphocytes and NK cells and application for in vivo bio-imaging. In Vivo. 2014; 28(5):779-84.

18. Shekhar TM, Burvenich IJG, Harris MA, et al. Smac mimetics LCL161 and GDC-0152 inhibit osteosarcoma growth and metastasis in mice. BMC Cancer. 2019;19:924.

19. Cardiff RD, Miller CH, Munn RJ. Manual hematoxylin and eosin staining of mouse tissue sections. Cold Spring Harb Protoc. 2014;2014(6):655-8.

20. Berlin O, Samid D, Donthineni-Rao R, et al. Development of a novel spontaneous metastasis model of human osteosarcoma transplanted orthotopically into bone of athymic mice. Cancer Res. 1993;53(20):4890-5.

21. Habu S, Fukui H, Shimamura K, et al. In vivo effects of anti-asialo GM1. I. Reduction of NK activity and enhancement of transplanted tumor growth in nude mice. J Immunol. 1981;127(1):34-8.

22. Nishikado H, Mukai K, Kawano Y, et al. NK cell-depleting anti-asialo GM1 antibody exhibits a lethal off-target effect on basophils in vivo. J Immunol. 2011;186(10):5766-71.

23. Coupland LA, Chong $\mathrm{BH}$, Parish CR. Platelets and P-selectin control tumor cell metastasis in an organ-specific manner and independently of NK cells. Cancer Res. 2012; 72(18):4662-71.

24. Schwartz C, Eberle JU, Voehringer D. Basophils in inflammation. Eur J Pharmacol. 2016;778:90-5.

25. Kiany S, Huang G, Kleinerman ES. Effect of entinostat on NK cell-mediated cytotoxicity against osteosarcoma cells and osteosarcoma lung metastasis. Oncoimmunology. 2017;6(8):e1333214.

26. Nishi T, Kusumi T, Tanaka M, et al. Establishment of transplantable murine osteosarcoma cell line with endochondral ossification. Anticancer Res. 2008;28(3a):1627-31.

27. Jia SF, Worth LL, Kleinerman ES. A nude mouse model of human osteosarcoma lung metastases for evaluating new therapeutic strategies. Clin Exp Metastasis. 1999;17(6):501-6.

28. Nakano T, Tani M, Ishibashi Y, et al. Biological properties and gene expression associated with metastatic potential of human osteosarcoma. Clin Exp Metastasis. 2003;20(7):665-74.

29. Nanni P, Landuzzi L, Manara MC, et al. Bone sarcoma patient-derived xenografts are faithful and stable preclinical models for molecular and therapeutic investigations. Sci Rep. 2019;9(1):12174.

30. Stewart E, Federico SM, Chen X, et al. Orthotopic patientderived xenografts of paediatric solid tumours. Nature. 2017;549(7670):96-100.

31. Alzubi MA, Turner TH, Olex AL, et al. Separation of breast cancer and organ microenvironment transcriptomes in metastases. Breast Cancer Res. 2019;21(1):36.

32. Sprouffske K, Kerr G, Li C, et al. Genetic heterogeneity and clonal evolution during metastasis in breast cancer patient-derived tumor xenograft models. Comput Struct Biotechnol J. 2020;18:323-31.

Address correspondence to: Christine J. Hawkins, PhD Department of Biochemistry and Genetics La Trobe Institute for Molecular Science La Trobe University Kingsbury Drive, Bundoora 3086 Victoria Australia

Email: c.hawkins@latrobe.edu.au 